BMJ Open Sport \& Exercise Medicine

\title{
Observable player behaviours and playing performance following helmet strikes in elite cricket
}

\author{
Patrick O'Halloran (D) ,, ${ }^{1,2}$ Luke Goggins (1) , ${ }^{3}$ Nicholas Peirce (D) ${ }^{4,5}$
}

To cite: 0 'Halloran $P$, Goggins $L$, Peirce N. Observable player behaviours and playing performance following helmet strikes in elite cricket. BMJ Open Sport \& Exercise Medicine 2021;7:e01128. doi:10.1136/ bmjsem-2021-001128

- Additional supplemental material is published online only. To view, please visit the journal online (http://dx.doi. org/10.1136/bmjsem-2021 001128)

Accepted 22 0ctober 2021

\section{Check for updates}

(c) Author(s) (or their employer(s)) 2021. Re-use permitted under CC BY-NC. No commercial re-use. See rights and permissions. Published by BMJ.

${ }^{1}$ Marker Diagnostics UK Ltd, Birmingham, UK

${ }^{2}$ Musculoskeletal Medicine, Royal Orthopaedic Hospital NHS Foundation Trust, Birmingham, UK

${ }^{3}$ Department for Health, University of Bath, Bath, UK ${ }^{4}$ Science and Medicine, England and Wales Cricket Board, Loughborough, UK

${ }^{5}$ National Centre for Sports Medicine, Loughborough University, Loughborough, UK

Correspondence to Dr Patrick O'Halloran; patrickohalloran@doctors.org.uk

\section{ABSTRACT}

Objectives Investigate the observable player behaviours and features of both concussive (HS-C) and nonconcussive (HS-NC) helmet strikes and describe their impact on playing performance.

Methods Elite male cricketers sustaining helmet strikes between the 2016 and 2018 seasons were identified by the England and Wales Cricket Board. Medical records identified players sustaining a concussion and those in whom concussion was excluded. Retrospective cohort analysis was performed on batting and bowling performance data available for these players in the 2 years prior to and 3 months post helmet strike. Video analysis of available incidents was conducted to describe the characteristics of the helmet strike and subsequent observable player behaviours. The HS-C and HS-NC cohorts were compared.

Results Data were available for 194 helmet strikes. 56 $(29 \%)$ resulted in concussion. No significant differences were seen in playing performance in the 3 months post concussive helmet strike. However, a significant decline in batting performance was seen in this period in the HS-NC group $(\mathrm{p}<0.001)$.

Video features signifying motor incoordination were most useful in identifying concussion post helmet strike, however, typical features suggesting transient loss of consciousness were not seen. Features such as a longer duration pause prior to the batsman resuming play and the level of concern shown by other players were also useful features.

Conclusion HS-NC may be more significant for player performance than previously thought. Guidance for using video replay to identify concussion in cricket may need to be modified when compared with other field sports.

\section{INTRODUCTION}

Head impacts occur in elite cricket at a rate of 7.2 per 1000 player days with concussions occurring at 2.3 per 1000 player days. Although the risk of both is not uniformly spread through the team, this equates to one player receiving a head impact every 12 days of play and a concussion every 36 days of play, per team. ${ }^{1}$

These injuries are a source of concern. Partly, this relates to research linking concussive head injuries to long-term psychiatric and

\section{Key messages}

What is already known

- Helmet strikes are common in cricket and are a source of concern in both the short and long term.

- Approximately, $70 \%$ of concussions occurring after such impacts are not diagnosed immediately demonstrating the need to understand their recognition better.

- Sporting performance can be impaired following sporting head injury in other codes.

What are the new findings

- Helmet strikes that result in concussion do not appear to significantly impact batting or bowling performance post return to play in elite cricket.

- However, helmet strikes which do not result in concussion do appear to result in significant batting performance impairment for up to 3 months post impact.

- Observable features and player behaviours of concussed players following helmet strike appear to differ from those seen in other sports and this should be widely disseminated to aid concussion detection in cricket.

neurodegenerative disease, although Jones $e t$ $a l^{2}$ reported no increased risk of dementia in retired professional cricketers. However, there is also the potential for impaired playing performance, increased musculoskeletal injury risk and, in elite sport, limitation of career length and earning potential in the short- and medium-term following concussion. ${ }^{3-7}$

There are also concerns around repetitive 'sub-concussive' impacts and the potential for these accumulated impacts to be as important in the short- and long-term brain health of athletes. $^{8-10}$

Improved understanding of head impact injuries in cricket is important, particularly given approximately $70 \%$ of concussions occurring after such injuries are not diagnosed at the time of the strike. Possibly, this may be because the 'traditional' observable features signifying transient loss of consciousness seen 
after concussion in other sporting codes are not common in the elite cricket setting. ${ }^{111}$

Thus, our objective was to investigate helmet strikes occurring in elite English cricket from two perspectives, to inform the detection of significant strikes and the aftercare of players suffering them. First, to describe the observable player behaviours seen on video review following helmet strike and analyse any differences seen between players subsequently diagnosed with concussion versus those in whom concussion is excluded. Second, to describe effects on playing performance following helmet strike and, again, differences seen based on concussion diagnosis.

\section{METHODS}

\section{Study design}

Helmet strikes were defined as the ball hitting the batsman's helmet, via any mechanism, be that directly from the bowler, from the throw of a fielding player, etc. Players sustaining helmet strikes between 2016 and 2018 seasons while playing in matches, where video recording and documentation are mandated (First Class County and International), were identified through video analysis supplied by England and Wales Cricket Board (ECB) Performance Analysts.
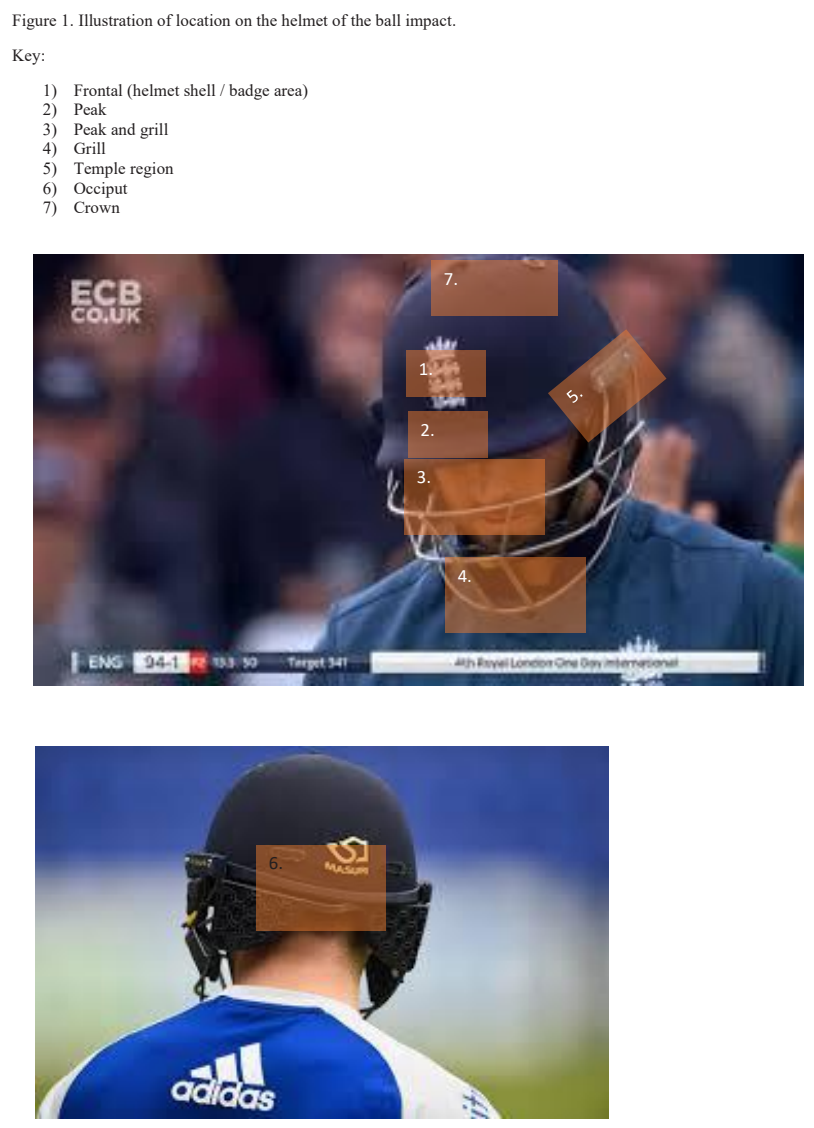

igure 1 Illustration of location on the helmet of the ball impact. Key: 1. frontal (helmet shell / badge area); 2. peak; 3. peak and grill; 4. grill; 5. temple region; 6. occiput; 7 . crown.
Concussion injuries were recorded within the ongoing ECB injury surveillance programme, which is centrally coordinated by an 'Injury Surveillance Officer' (ISO) who monitors compliance and injury definitions. The diagnosis of concussion was made by the doctor and physiotherapist at each team according to the ECB and International Cricket Council Concussion Guidelines, written with reference to the most recent Concussion in Sport Group Consensus Statements at the time. The data collection spans two Consensus Statements. ${ }^{4} 1213$

All injuries were recorded on purpose built central online medical records systems: Profiler (The Profiler Corporation, New Zealand (2016)), Cricket Squad (The Sports Office, UK (2017-2018 inclusive)), supported by ECB's ISO. To improve compliance, the ECB mandates consistent standards for injury and medical record keeping for the domestic game through annual Science and Medicine Audits.

A retrospective cohort analysis was used to identify players who received a helmet strike and sustained a concussion (HS-C) or received a helmet strike and did not sustain a concussion (HS-NC) during the study period. Concussions occurring without video documentation were excluded.

\section{Performance analysis}

ECB Performance Analysts recorded player batting and bowling performance data for each competitive game on a purpose built central online system. Bowling performance data were analysed to separate the potential effects of changes in player behaviour following helmet strike versus changes in, for example, reaction time or motor speed.

Batting and bowling averages were obtained for 2 years prehelmet strike (from date of injury/incident), 1 and 3 months post return to play (RTP). An inclusion criterion for performance data was set with batting and bowling averages for first XI First-Class domestic County Championship cricket fixtures being included, where the player had participated in at least two innings (for batting) or overs (for bowling). First-Class County Championship performance was selected over the two other competition formats (One-Day 50 over and T20 cricket) as fixtures span the whole domestic season (from April to September inclusive), providing more opportunity to get enough performance data from fixtures that meet the inclusion criteria for the various time periods. In comparison, the other domestic competition formats (One-Day and T20) are played in intermittent 'blocks' of fixtures over a month or so. Due to the timing of incidents, complete data for each player at each time period were not always available.

\section{Video analysis}

The ECB ISO collated all available videos of helmet strikes at First-Class County teams (across County Championship, One Day 50 Over and T20 cricket) and the men's senior national side from each team's Performance 
Frontal Plane

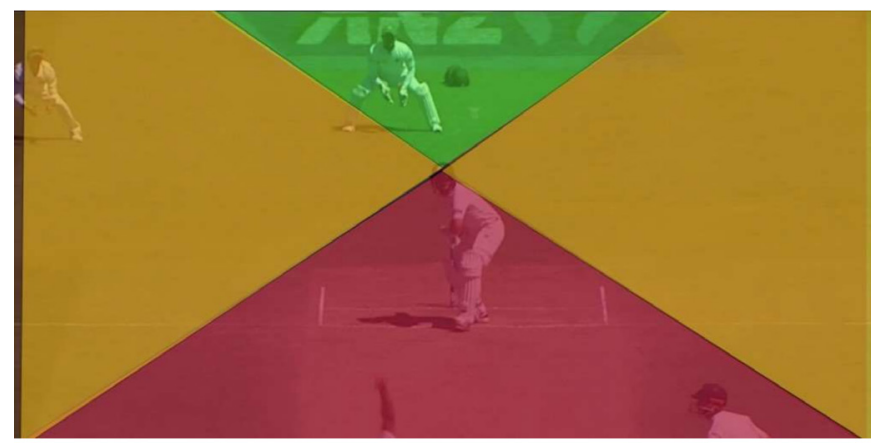

Sagittal Plane

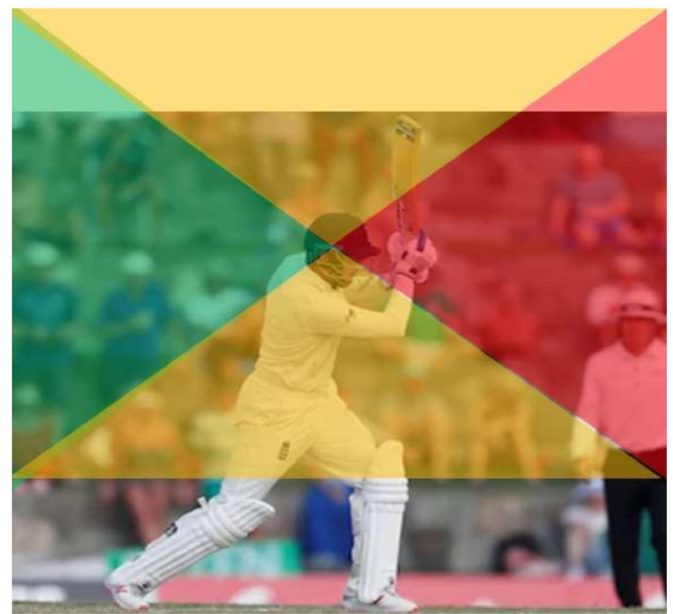

Figure 2 Illustration of the direction of ball travel following helmet strike.

Analyst. A selection of these videos was reviewed by the ISO and the lead author to identify visible features that may be relevant to investigate further in the entire cohort of videos.

This review was made with reference to the Berlin Consensus Statement, the concussion protocols of the ICC, World Rugby, FIFA and the consensus statement on video features of concussion. This produced a list of 19 signs the player may display and three variables relating to the impact location and subsequent direction of the ball (figures 1 and 2).

The ball directions were coded as red, yellow and green corresponding to the theoretical transfer of energy in each. For example, if the ball strikes the helmet and continues behind the batsman, this is likely to represent a glancing blow (green) as compared with the ball striking the helmet and travelling directly back down the pitch (red). This list was reviewed by three experienced ECB doctors with only minor changes suggested. $^{411}{ }^{13-16}$ The final list is included in online supplemental appendix 1.

All videos were then reviewed and coded by two researchers. Any discrepancies were discussed and if consensus could not be reached, the senior author was consulted. The mean of the two transverse plane ball angles was calculated.

\section{Statistical analysis}

Performance analysis

Batting and bowling averages were converted to withinindividual z-scores and summarised with descriptive statistics before the assumption of normality was assessed with the Shapiro-Wilks test. Due to the variation in data available for each player across each time period, mean differences between the three performance time periods were assessed, with either a one-way Analysis of Variance (ANOVA) (when normality can be assumed) or KruskalWallis (when normality cannot be assumed), along with suitable post hoc tests and 'Benjamini-Hochberg' adjusted $\mathrm{p}$ values. All estimations were made using the lme4 package with R (V.3.5.2, R Foundation for Statistical Computing, Vienna, Austria). ${ }^{17}$

\section{Video analysis}

Descriptive statistics were calculated for each feature. $\chi^{2}$ test was used to compare the prevalence of each feature between the HS-C and HS-NC groups. Location of impact and ball direction after impact were assessed both alone and in combination. For these features, relative risk of subsequent concussion diagnosis was calculated. For the transverse plane ball angle, assumption of normality was assessed with Shapiro-Wilks test and then either t test or Mann-Witney U used to assess for differences between the groups. The sensitivity and specificity of video features relating to player behaviour were assessed for predicting subsequent concussion diagnosis.

\section{Patient and public involvement}

Patients and/or the public were not involved in the design, or conduct, or reporting, or dissemination plans of this research.

\section{RESULTS}

194 male senior players, registered to compete in the ECB domestic First-Class County Championship from 2016 to 2018 inclusive, received a helmet strike, 56 HS-C and 153 HS-NC.

\section{Performance analysis}

Batting performance

Adequate batting performance data were available for 24 of the 56 HS-C group and 138 of the 153 HS-NC group. Performance data were not available for each player for each time period. A summary of the available data is shown in table 1 , with the individual raw batting averages for each group in online supplemental tables 1 and 2 .

The mean, within-individual, batting performance z-scores for the HS-C group increased from 2-year prehelmet strike to 1-month post-RTP before decreasing between 1-month post and 3-month post-RTP (figure 3). In the HS-NC group, mean within-individual performance decreased from 2-year pre to 1-month post incident and then decreased further from 1-month to 3-month post incident (figure 3). 
Table 1 Summary of the number of players with available batting and bowling performance data points, following helmet strike for each time period between the HS-C and HS-NC groups

\begin{tabular}{lll}
\hline Batting performance & HS-C & HS-NC \\
\hline 2 years prestrike & 24 & 138 \\
1-month poststrike & 16 & 116 \\
3-month poststrike & 22 & 105 \\
Bowling performance & & \\
2 years prestrike & 12 & 58 \\
1-month poststrike & 5 & 49 \\
3-month poststrike & 10 & 42 \\
\hline
\end{tabular}

HS-C, helmet strike-concussion; HS-NC, helmet strike-no concussion.

For the HS-C group, the assumption of normality was not met ( $\mathrm{W}=0.88, \mathrm{p} \leq 0.001)$, and no difference in batting performance was seen between the three time periods $(\mathrm{H}(2)=1.58, \mathrm{p}=0.45)$.

For the HS-NC group, the assumption of normality was also not met $(\mathrm{W}=0.88, \mathrm{p}=<0.001)$. A difference in batting
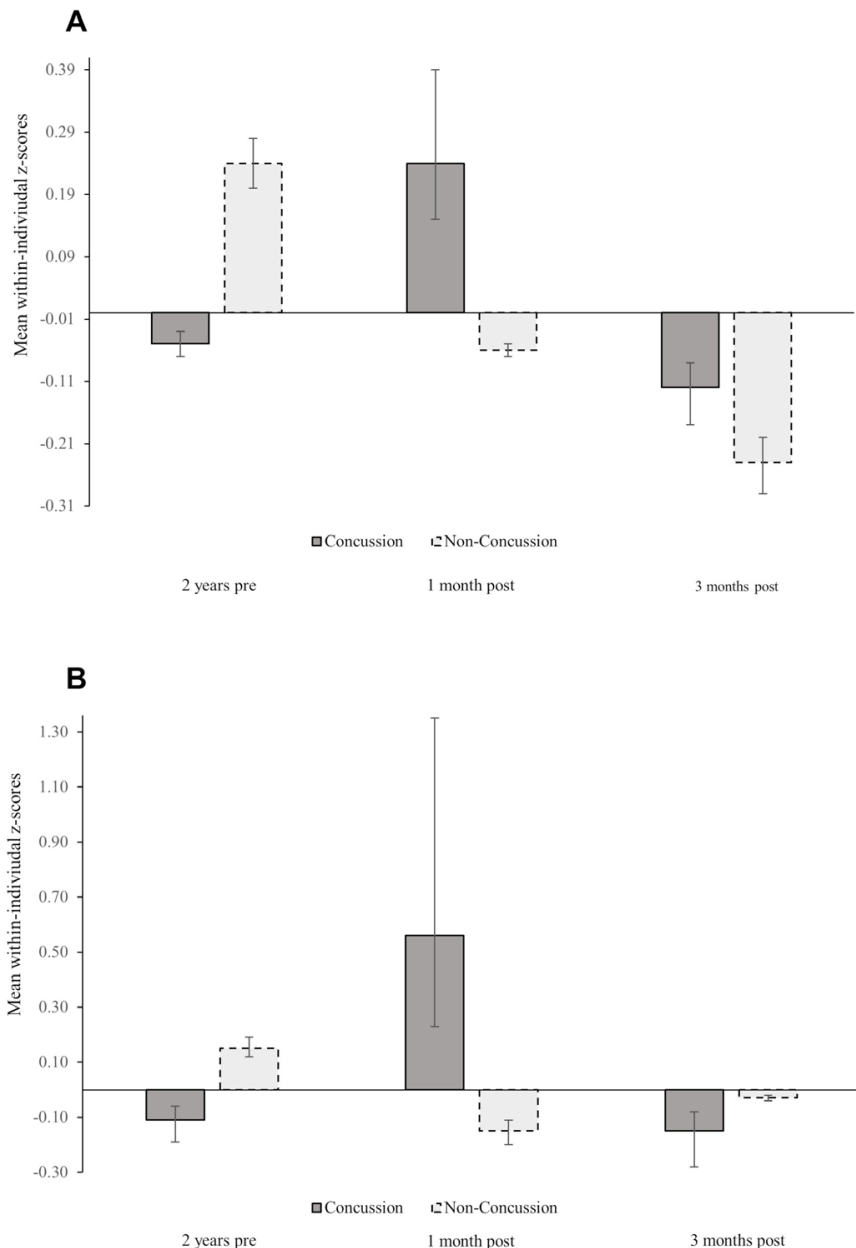

Figure 3 Mean within-individual performance z-scores for 2 years pre-helmet strike to 1 and 3 month post return to play for batting $(A)$ and bowling $(B)$. performance was seen between the three time periods $(\mathrm{H}(2)=21.48, \mathrm{p}<0.001)$. Post hoc tests were conducted using Wilcoxon rank sum tests and found differences in batting performance with a small effect for 2 years pre and 1 month post $(\mathrm{p}<0.05, r=0.17)$ and a moderate effect for 2 years pre to 3 months post $(\mathrm{p}<0.001, r=0.31)$. No differences were found between 1 and 3 months post-RTP $(\mathrm{p}=0.16, \mathrm{r}=0.09)$.

Therefore, following a HS-NC, there was a reduction in batting average from 2 years pre incident (mean $=0.24 \pm 0.75$ ) to 1 month post (mean $=-0.06 \pm 0.83$ ) with a continued reduction in batting performance 3 months post incident (mean $=-0.24 \pm 0.70)$.

\section{Bowling performance}

Adequate data for bowling performance were available for 12 of the HS-C group and 58 of the HS-NC group. A summary of the available data is provided in table 1 , with the individual raw bowling averages for each group in online supplemental tables 3 and 4 .

The mean within-individual bowling performance z-scores for the HS-C group followed a similar pattern to the batting performance, increasing from 2-year prehelmet strike to 1-month post-RTP before decreasing from 1-month to 3-month post-RTP (figure 3). For those in the HS-NC group, mean within-individual bowling performance z-scores decreased from 2 years pre to 1 month post before recovering slightly from 1 month to 3 months post but still down from their performance 2 years pre (figure 3 ).

For the HS-C group, the assumption of normality was not met $(\mathrm{W}=0.80, \mathrm{p}=<0.001)$, and no difference in bowling performance between the three time periods was seen $(\mathrm{H}(2)=3.00, \mathrm{p}=0.22)$. Similarly, for HS-NC group, the assumption of normality was not met $(\mathrm{W}=0.88$, $\mathrm{p}=<0.001$ ), and no difference in bowling performance between the three time periods was seen $(\mathrm{H}(2)=3.04$, $\mathrm{p}=0.22)$.

\section{Video analysis}

Video was available from 169 helmet strikes, 16 HS-C versus 153 HS-NC. Table 2 demonstrates the distribution of features regarding location of impact and ball direction. When assessing the location of the ball impact, it was difficult on the views available to distinguish between the Frontal, Peak and Peak and Grill regions and, therefore, these were combined for further analysis. The relative risk of sustaining a concussion was found to be highest in this combined region (RR 2.26) followed by the grill alone and temple.

The most common direction of ball travel poststrike was into the yellow zone. This was also the zone with the highest relative risk of sustaining a concussion (RR 1.26). No relationship was found regarding transverse plane ball angle ( $\mathrm{U}=1080 \mathrm{p}=0.53)$.

To investigate these features further, their combined effects were analysed. This demonstrated that the greatest relative risk is seen in strikes, which impact on 
Table 2 Distribution of features related to location of impact of ball strike and subsequent ball direction poststrike between the HS-C and HS-NC groups

\begin{tabular}{|c|c|c|c|}
\hline & $\begin{array}{l}\text { Frequency } \\
\text { HS-C } \\
\mathrm{N}=16\end{array}$ & $\begin{array}{l}\text { Frequency } \\
\text { HS-NC } \\
\mathrm{N}=153\end{array}$ & Relative risk \\
\hline \multicolumn{4}{|c|}{$\begin{array}{l}\text { Position of helmet } \\
\text { strike }\end{array}$} \\
\hline Frontal & $318.8 \%$ & $2113.7 \%$ & \\
\hline Peak & $531.3 \%$ & $95.9 \%$ & \\
\hline Peak and grill & $16.3 \%$ & $2617 \%$ & $\begin{array}{l}\text { Combined } \\
\text { frontal, peak } \\
\text { and peak and } \\
\text { grill } 2.26\end{array}$ \\
\hline Grill & $318.8 \%$ & $2919 \%$ & 1.02 \\
\hline Temple & $318.8 \%$ & $4932 \%$ & 0.53 \\
\hline Occiput & 0 & $53.3 \%$ & \\
\hline Crown & 0 & $95.9 \%$ & \\
\hline Missing & 1 & 5 & \\
\hline \multicolumn{4}{|c|}{$\begin{array}{l}\text { Direction of ball } \\
\text { travel post helmet } \\
\text { strike }\end{array}$} \\
\hline Red & $531.3 \%$ & $5033.1 \%$ & 0.83 \\
\hline Yellow & $850 \%$ & $5637.1 \%$ & 1.26 \\
\hline Green & $318.75 \%$ & $3523.2 \%$ & 0.93 \\
\hline Missing & 0 & 12 & \\
\hline
\end{tabular}

HS-C, helmet strike-concussion; HS-NC, helmet strike-no concussion .

the combined frontal/peak regions and rebound into the green zone (RR 2.27) as well as those which impact on the grill region and rebound into the yellow zone (RR 2.05). Further details are provided in online supplemental appendix 2.

Table 3 demonstrates the player behaviour features seen most commonly following helmet strike in the HS-C. and HS-NC groups. The most sensitive features to distinguish HS-C were the player removing or checking their helmet $(92.31 \%)$ and longer duration of recovery time to face the next delivery $(91.67 \%)$. Meanwhile, the most specific features covered a range of behaviours relating to the responses of other participants on the field and the player's balance. During the video review, there was insufficient evidence to establish if the player retired from play following the incident or if medical staff entered the field of play uninvited, and so these were excluded.

\section{DISCUSSION}

We have attempted to describe head impact injuries in elite cricket from the perspective of both their observable characteristics and their impacts on player performance in the context of concussive and 'subconcussive' injuries.

These findings indicate a non-significant trend towards initial increased batting and bowling performance at 1-month poststrike, following by a decline in performance at 3 months poststrike in those who suffer a helmet strike with concussion. This is consistent with findings from elite baseball by Sabesan et $a l^{18}$ which showed a non-significant decline in batting and pitching performance on RTP postconcussion. However, this contrasts with Wasserman et $a l \mathrm{~s}^{19}$ work in the same population. They demonstrated a significant reduction in batting performance in the initial 2 weeks post-RTP, with a nonsignificant reduction at 4-6 weeks. ${ }^{18} 19$

Studies on playing performance in other sports postconcussion, however, provide similarly inconsistent results, even within the same sport. A possible explanation for this may be the management of RTP following concussion. In elite soccer, Ramkumar et al demonstrated that players returning through more aggressive protocols suffered a decrement in performance versus those managed more conservatively.

Crucially, however, a significant reduction in batting performance was seen following a helmet strike, which did not result in concussion at both 1 and 3 months. While reductions in motor control have been described following subconcussive impacts in boxers, the effect of such impacts on 'real-world' sports performance is not well studied. ${ }^{20}$

Possibly, this may reflect concussions, which are not detected under the current approach to diagnosis. Alternatively, the differences seen between the HS-C and HS-NC groups may be explained by the extended period of rest and graded recovery afforded by the concussion diagnosis. Whether this acts to ameliorate a lasting effect on neurocognitive/motor performance or an independent effect on player psychology and behaviour should also be considered. This is important to investigate further as it may highlight a need for further assessment of these players and a managed RTP, even in the absence of concussion.

Our findings also suggest that helmet strikes occurring to the frontal, peak and grill sections of the helmet represent the greatest relative risk in terms of subsequent diagnosis of concussion. This contrasts with accelerometry and modelling data from other helmeted and non-helmeted sports that suggest lateral impacts are more injurious. ${ }^{21-23}$ In cricket, where the bowling line is permitted to contact the batsman who must react, impacts to the front of the helmet may result from a faster ball speed while a slower ball on a similar trajectory may prompt a player to turn their head in preparation for an impact. However, this relationship is clearly complex, as demonstrated by Saw et al, ${ }^{24}$ who's work highlighted that impacts to the back of the helmet carried the highest positive predictive value of concussion.

Alternatively, the combination of helmet strike location and the subsequent direction of ball travel could be important. Counter to our intuitive colour-coding zones, impacts in the Yellow zone represented the largest concussion risk. This may be because, when the ball strikes the frontal section and rebounds laterally (Yellow zone), the rotational force on the head is greater. Rotational, rather than translational, impacts are theorised to 
Table 3 Player behaviour features and diagnostic performance characteristics following helmet strike between the HS-C and HS-NC groups

\begin{tabular}{|c|c|c|c|c|c|}
\hline Player behaviour feature & $\begin{array}{l}\text { Frequency } \\
\text { HS-C } \\
\mathrm{N}=16\end{array}$ & $\begin{array}{l}\text { Frequency HS- } \\
\mathrm{NC} \\
\mathrm{N}=153\end{array}$ & $\chi^{2}$ with $p$ values & $\begin{array}{l}\text { Sensitivity } \\
(\%)\end{array}$ & $\begin{array}{l}\text { Specificity } \\
(\%)\end{array}$ \\
\hline Player follows ball after strike & 5 & 63 & $0.63 p-0.59$ & 31.25 & 58.56 \\
\hline Player removes or checks helmet & 12 & 56 & $13.01 p-<0.001$ & 92.31 & 59.71 \\
\hline Other players - relaxed & 7 & 57 & $0.34 p-0.58$ & 50 & 58.09 \\
\hline Other players-concerned & 0 & 11 & $1.21 \mathrm{p}-0.6$ & 0 & 91.97 \\
\hline Other players-urgent & 5 & 11 & $10.28 p-0.01$ & 35.71 & 91.97 \\
\hline Player stumbles & 4 & 32 & $0.11 p-0.75$ & 33.33 & 78.67 \\
\hline Player falls to knees & 2 & 12 & $0.40 p-0.63$ & 12.5 & 92.11 \\
\hline Player falls over & 3 & 7 & $5.23 p-0.06$ & 18.75 & 95.42 \\
\hline Player stumbles getting back to feet & 0 & 2 & $0.20 p-1.0$ & 0 & 98.62 \\
\hline Walks away from play & 4 & 64 & $0.53 p-0.56$ & 33.33 & 55.86 \\
\hline Player holds face/head & 4 & 19 & $2.71 p-0.11$ & 30.77 & 86.33 \\
\hline Player bends head down & 7 & 36 & $3.73 p-0.07$ & 50 & 94.55 \\
\hline Player immediately alert & 4 & 67 & $2.63 p-0.12$ & 25 & 53.79 \\
\hline Play resumes immediately & 1 & 56 & $6.48 p-0.01$ & 6.67 & 60 \\
\hline Player appears dazed & 5 & 16 & $5.05 p-0.04$ & 50 & 81.18 \\
\hline More than $4 \mathrm{~s}$ to resume & 11 & 61 & $8.13 p-0.01$ & 91.67 & 51.59 \\
\hline Player knocked backwards & 3 & 43 & $0.66 p-0.56$ & 18.75 & 71.71 \\
\hline
\end{tabular}

HS-C, helmet strike-concussion; HS-NC, helmet strike-no concussion.

be more likely to result in concussion. This also raises a question about whether neck strengthening interventions may be beneficial for primary prevention. ${ }^{24} 25$

Green zone impacts were also found to have higher concussion risk than expected, however, our suspicion is that this represents difficulties in judging the flight of the ball in the frontal plane. Frequently, only one view was available, usually in the frontal plane and often too narrow to view the landing of the ball after rebound. Therefore, travel into the green zone on the frontal view may in fact appear in the red zone in the sagittal view, suggesting diagnosis could be improved with access to multiple angles. This is supported by the findings of Saw $e t a l^{44}$ who conducted a similar exercise in Australian cricket and demonstrated that the rebound of the ball towards the source or the ball stopping dead was associated with a higher risk of concussion. Standardisation of the views and reference frame in future may assist in investigating this further.

The ECB requires all players to undergo annual baseline testing using the Standardized Concussion Assessment Tool 5 and Immediate Post-Concussion Assessment and Cognitve Test (ImPACT) (ImPACT Applications, Coralville) on a biannual basis unless helmet strikes are recorded, in which case it is annual. This data suggest that more detailed ImPACT testing should continue and that annual reviews should be considered. Jones et $a t^{2}$ surveyed retired cricketers for long-term health outcomes. No cases of dementia/neurocognitive disease were recorded, but ongoing review of retired cricketers and any potential consequence of long-term helmet strikes is warranted.

Notably, observable signs of concussion in this context appear different to those from other field sports. The value of video replay in detecting concussion is clear, particularly in football codes, and the features, which suggest a transient loss of consciousness or a concussion, are well documented. However, none of those features appeared in this series and their absence may make concussion diagnosis more challenging in cricket if this is not recognised. ${ }^{111152627}$

Features suggesting motor incoordination were valuable in detecting concussion in this study. However, features indicating urgency and concern from other players or officials as well as the player inspecting their helmet and pausing for $>4 \mathrm{~s}$ before the next delivery were also important. These may be unique to cricket where rules and culture dictate the action pauses until the batsman is ready.

These findings suggest several areas for further investigation, both to understand their implications and to improve player safety. However, some important limitations should be recognised. First, our groups were weighted towards HS-NC. As a result, the concussive helmet strike element of the performance analysis was underpowered, which may explain the lack of significant findings in this group. This was unavoidable in view of the low incidence of concussive helmet strikes in 
cricket; however, it is an important caveat. In addition, we excluded from our analysis head impacts occurring to fielding or bowling players. This was due to the low total number of these incidents, all of them resulting in concussion. Finally, we were not able to analyse differences in the clinical status of the player between those helmet strikes captured on video and those which could not be reviewed, which may introduce a potential source of bias, though we feel this is unlikely.

\section{CONCLUSION}

Helmet strikes are common in elite cricket. While the rate of concussion following helmet strike is low, these findings suggest that HS-NC may be more important than previously thought and may require management involving a supported RTP. For example, involving consideration of and interventions directed at visual motor speed and psychological readiness. The video features of helmet strikes, which may point to concussion, appear to differ in cricket when compared with other sports and this requires further investigation and dissemination to ensure that medical staff are able to recognise key features in the cricket context.

Acknowledgements The authors would like to thank the ECB Injury Surveillance Officers, Orla Purdon, Rachel Taggart and Olivia Hellas for their assistance in completing this project. We would also like to thank Dr Gurjit Bhogal and Dr Thamindu Wedatilake for their review of the proposed video criteria. In addition, we would like to thank Isabella Blair of the University of Birmingham for her assistance in coding the video footage. Finally, we wish to thank all of the medical and support staff at participating teams for the collection of the data used in this study.

Contributors All three authors were involved in the design of the study and the evaluation and interpretation of the results. $\mathrm{PO}$ 'H prepared the manuscript. LG performed the data analysis on the playing performance data and $\mathrm{PO}$ 'H performed the analysis on the video review data. $\mathrm{PO}$ 'H was responsible for the overall content as the guarantor. All three authors were involved in reviewing and editing the manuscript.

Funding Dr PO'H has received funding from The Drake Foundation.

Competing interests None declared.

Patient consent for publication Not applicable.

Ethics approval This research was approved by the ECB Research Committee.

Provenance and peer review Not commissioned; externally peer reviewed.

Data availability statement Data are available upon reasonable request. Deidentified summary data can be made available by the lead author upon reasonable request.

Open access This is an open access article distributed in accordance with the Creative Commons Attribution Non Commercial (CC BY-NC 4.0) license, which permits others to distribute, remix, adapt, build upon this work non-commercially, and license their derivative works on different terms, provided the original work is properly cited, appropriate credit is given, any changes made indicated, and the use is non-commercial. See: http://creativecommons.org/licenses/by-nc/4.0/.

\section{ORCID iDs}

Patrick 0'Halloran http://orcid.org/0000-0002-1185-3485

Luke Goggins http://orcid.org/0000-0001-5540-3729

Nicholas Peirce http://orcid.org/0000-0002-3272-5060

\section{REFERENCES}

1 Hill T, Orchard J, Kountouris A. Incidence of concussion and head impacts in Australian Elite-Level male and female cricketers after head impact protocol modifications. Sports Health 2019;11:180-5
2 Jones ME, Davies MAM, Leyland KM, et al. Osteoarthritis and other long-term health conditions in former elite cricketers. J Sci Med Sport 2018;21:558-63.

3 Orchard JW, Ranson C, Olivier B, et al. International consensus statement on injury surveillance in cricket: a 2016 update. $B r \mathrm{~J}$ Sports Med 2016;50:1245-51.

4 McCrory P, Meeuwisse W, Dvořák J, et al. Consensus statement on concussion in sport-the $5^{\text {th }}$ international conference on concussion in sport held in Berlin, October 2016. Br J Sports Med 2017:51:838-47.

5 McPherson AL, Nagai T, Webster KE, et al. Musculoskeletal injury risk after sport-related concussion: a systematic review and metaanalysis. Am J Sports Med 2019;47:1-8.

6 Navarro SM, Sokunbi OF, Haeberle HS, et al. Short-Term outcomes following concussion in the NFL: a study of player longevity, performance, and financial loss. Orthop J Sports Med 2017;5:1-7.

7 Ramkumar PN, Navarro SM, Haeberle HS, et al. Concussion in American versus European professional soccer: a decade-long comparative analysis of incidence, return to play, performance, and longevity. Am J Sports Med 2019;47:2287-93.

8 Mackay DF, Russell ER, Stewart K, et al. Neurodegenerative disease mortality among former professional soccer players. N Engl J Med 2019;381:1801-8.

9 LoBue C, Schaffert J, Cullum CM. Chronic traumatic encephalopathy: understanding the facts and debate. Curr Opin Psychiatry 2020;33:130-5.

10 Manley G, Gardner AJ, Schneider KJ, et al. A systematic review of potential long-term effects of sport-related concussion. Br J Sports Med 2017:51:969-77.

11 Davis GA, Makdissi M, Bloomfield P. International consensus definitions of video review of concussion in professional sports. $\mathrm{Br} \mathrm{J}$ Sports Med 2018:1-4

12 McCrory P, Meeuwisse W, Aubry M, et al. Consensus statement on concussion in sport--the 4th International Conference on Concussion in Sport held in Zurich, November 2012. Clin J Sport Med 2013;23:89-117.

13 International Cricket Council. Concussion management guidelines, 2018. Available: http://bjsm.bmj.com/content/47/5/250 [Accessed 8 Apr 2021].

14 Kramer E, Dvořák J. Fifa football emergency medical manual. 2nd Edition. FIFA, 2015.

15 Fuller GW, Kemp SPT, Raftery M. The accuracy and reproducibility of video assessment in the pitch-side management of concussion in elite rugby. J Sci Med Sport 2017;20:246-9.

16 Davis G. Makdissi M. Use of video to facilitate sideline concussion diagnosis and management decision-making. J Sci Med Sport 2016;19:898-902.

17 Bates D, Mächler M, Bolker B, et al. Fitting Linear Mixed-Effects Models Using Ime4. J Stat Softw 2015;67.

18 Sabesan VJ, Prey B, Smith R, et al. Concussion rates and effects on player performance in major league baseball players. Open Access $J$ Sports Med 2018;9:253-60.

19 Wasserman EB, Abar B, Shah MN, et al. Concussions are associated with decreased batting performance among major league baseball players. Am J Sports Med 2015;43:1127-33.

20 Di Virgilio TG, letswaart M, Wilson L, et al. Understanding the consequences of repetitive Subconcussive head impacts in sport: brain changes and dampened motor control are seen after boxing practice. Front Hum Neurosci 2019;13:294.

21 Elkin BS, Gabler LF, Panzer MB, et al. Brain tissue strains vary with head impact location: a possible explanation for increased concussion risk in struck versus striking football players. Clinical Biomechanics 2019;64:49-57.

22 Liao S, Lynall RC, Mihalik JP. The effect of head impact location on day of diagnosed concussion in college football. Med Sci Sports Exerc 2016;48:1239-43.

23 McIntosh AS, Patton DA, Fréchède B, et al. The biomechanics of concussion in unhelmeted football players in Australia: a casecontrol study. BMJ Open 2014:4:5078.

24 Saw AE, Howard DJ, Kountouris A, et al. Situational factors associated with concussion in cricket identified from video analysis. J Concussion 2020;4:205970022094719.

25 Kleiven S. Why most traumatic brain injuries are not caused by linear acceleration but skull fractures are. Front Bioeng Biotechnol 2013;1.

26 Collins CL, Fletcher EN, Fields SK, et al. Neck strength: a protective factor reducing risk for concussion in high school sports. J Prim Prev 2014:35:309-19.

27 Makdissi M, Davis G. The reliability and validity of video analysis for the assessment of the clinical signs of concussion in Australian football. J Sci Med Sport 2016;19:859-63. 